\title{
Exact Solution of Asymmetric Diffusion With Second-Class Particles of Arbitrary Size
}

\author{
F. C. Alcaraz ${ }^{1}$ R. Z. Bariev ${ }^{1,2}$ \\ ${ }^{1}$ Departamento de Física, \\ Universidade Federal de São Carlos, 13565-905, São Carlos, SP Brazil \\ ${ }^{2}$ The Kazan Physico-Technical Institute of the Russian Academy of Sciences, Kazan 420029, Russia
}

Received 15 November 1999

\begin{abstract}
The exact solution of the asymmetric exclusion problem with first- and scond-class particles is presented. In this model the particles (size 1) of both classes are located at lattice points, and diffuse with equal asymmetric rates, but particles in the first class do not distinguish those in the second class from holes (empty sites). We generalize and solve exactly this model by considering molecules in the first and second class with sizes $s_{1}$ and $s_{2}\left(s_{1}, s_{2}=0,1,2, \ldots\right)$, in units of lattice spacing, respectively. The solution is derived by a Bethe ansatz of nested type. We give a simple pedagogical presentation of the Bethe ansatz solution of the problem which can easily be followed by a reader not specialized in exactly integrable models.
\end{abstract}

\section{Introduction}

The similarity between the master equation describing time fluctuations in nonequilibrium problems and the Schrödinger equation describing the quantum fluctuations of quantum spin chains turns out to be fruitful for both areas of research [1] - [15]. Since many quantum chains are known to be exactly integrable through the Bethe ansatz, this provides exact information on the related stochastic model. At the same time the classical physical intuition and probabilistic methods successfully applied to nonequilibrium systems give new insights into the understanding of the physical and algebraic properties of quantum chains.

An example of this fruitful interchange is the problem of asymmetric diffusion of hard-core particles on the one dimensional lattice. This model is related to the exactly integrable anisotropic Heisenberg chain [16] (XXZ model). However if we demand this quantum chain to be invariant under a quantum group symmetry $U_{q}(S U(2))$, we have to introduce, for the equilibrium statistical system, unphysical surface terms, which on the other hand have a nice and simple interpretation for the related nonequilibrium stochastic system [3, 4].

In the area of exactly integrable models it is well known that one of the possible extensions of the spin$\frac{1}{2} \mathrm{XXZ}$ chain to higher spins, is the anisotropic spin-1 Sutherland model (grading $\epsilon_{1}=\epsilon_{2}=\epsilon_{3}=1$ ) [17]. On the other hand in the area of diffusion limited reactions a simple extension of the asymmetric diffusion prob- lem is the problem of diffusion with first and second class particles[18] - [20]. In this problem a mixture of two classes of hard-core particles diffuses on the lattice. Particles belonging to the first class ignore the presence of those in the second class, i. e., they see them in the same way as they see the holes (empty sites). In [3] it was shown that for open boundary conditions the anisotropic spin-1 Sutherland model and this last stochastic model are exactly related, the Hamiltonian governing the quantum or time fluctuations of both models being given in terms of generators of a Hecke algebra, invariant under the quantum group $U_{q} S U(3)$. In this paper we are going to derive the exact solution of the associated quantum chain, in a closed lattice. Recently [15] (see also [14]) we have shown that without losing its exact integrability, we can consider the problem of asymmetric diffusion with an arbitrary mixture of molecules with different sizes (even zero), as long they do not interchange positions, that is, there are no reactions. Motivated by these results we are going to extend the asymmetric diffusion problem with a second class of particles, to the case where the particles in each class have an arbitrary size, in units of the lattice spacing. Unlike the case of asymmetric diffusion problem, we have in this case a nested Bethe ansatz [21].

The paper is organized as follows. In the next section we introduce the generalized asymmetric model with second-class particles and derive the associated quantum chain. In section III the Bethe ansatz solution of the model is presented in a pedagogical and self 
contained way, which should be simple to follow by an audience not specialized in exactly integrable models. Finally in section IV we present our conclusions, with some possible generalizations of the stochastic problem considered in this paper, and some perspectives on future work.

\section{The generalized asymmetric diffusion model with first- and second-class of particles}

A simple extension of the asymmetric exclusion model, where hard-core particles diffuse on the lattice, is the problem where a mixture of particles belonging to different classes (first and second class) diffuses on the lattice. This problem was used to describe shocks [18] [20] out of equilibrium and also has a stationary probability distribution that can be expressed via the matrixproduct ansatz [22]. In this model we have $n_{1}$ and $n_{2}$ molecules belonging to the first and second class, respectively. Both classes of molecules diffuse asymmetrically, but with the same asymmetrical rates, whenever they encounter empty sites (holes) at nearest-neighbor sites. However, when molecules of different classes are at their minimum separation, the molecules of the first class exchange position with the same rate as they diffuse, and consequently the first-class molecules see no difference between molecules belonging to the second class and holes.

We now introduce a generalization of the above model, where instead of having unit size, the molecules in the first and second class have in general distinct sizes $s_{1}$ and $s_{2}\left(s_{1}, s_{2}=1,2, \ldots\right)$, respectively, in units of lattice spacing. In Fig. 1 we show some examples of molecules of different sizes. We may think of a molecule of size $s$ as formed by $s$ monomers (size 1), and for simplicity, we define the position of the molecule as the center of its leftmost monomer. The molecules have a hard-core repulsion: the minimum distance $d_{\alpha \beta}$, in units of the lattice spacing, between molecules $\alpha$ and $\beta$, with $\alpha$ in the left, is given by $d_{\alpha \beta}=s_{\alpha}$. In order to describe the occupancy of a given configuration of molecules we define, at each site $i$ of a lattice with $N$ sites, a variable $\beta_{i}(i=1,2, \ldots, N)$, taking the values $\beta_{i}=0,1$ and 2 , representing site $i$ empty ( size $s_{0}=1$ ), occupied by a molecule of class 1 ( $\operatorname{size} s_{1}$ ) or a molecule of class 2 ( $\left.\operatorname{size} s_{2}\right)$, respectively. Then the allowed configurations are given by the set $\left\{\beta_{i}\right\}(i=1, \ldots, N)$, where for each pair $\left(\beta_{i}, \beta_{j}\right) \neq 0$ with $j>i$ we should have $j-i \geq s_{\beta_{i}}$.

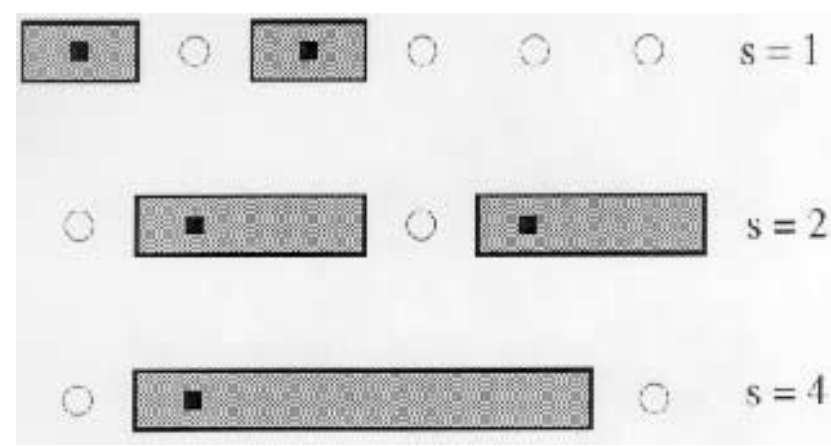

Figure 1. Example of configurations of molecules with distinct sizes $s$ in a lattice of size $N=6$. The coordinates of the molecules are denoted by the black squares.

The time evolution of the probability distribution $P(\{\beta\}, t)$, of a given configuration $\{\beta\}$ is given by the master equation

$$
\frac{\partial P(\{\beta\}, t)}{\partial t}=-\Gamma\left(\{\beta\} \rightarrow\left\{\beta^{\prime}\right\}\right) P(\{\beta\}, t)+\Gamma\left(\left\{\beta^{\prime}\right\} \rightarrow\{\beta\}\right) P\left(\left\{\beta^{\prime}\right\}, t\right),
$$

where $\Gamma\left(\{\beta\} \rightarrow\left\{\beta^{\prime}\right\}\right)$ is the transition rate for configuration $\{\beta\}$ to change to $\left\{\beta^{\prime}\right\}$. In the present model we only allow, whenever it is possible, the particles to diffuse to nearest-neighbor sites, or to exchange positions. The possible motions are diffusion to the right

$$
\begin{array}{ll}
1_{i} \emptyset_{i+s_{1}} \rightarrow \emptyset_{i} 1_{i+1}, & \left(\text { rate } \Gamma_{R}\right) \\
2_{i} \emptyset_{i+s_{2}} \rightarrow \emptyset_{i} 2_{i+1}, & \left(\text { rate } \Gamma_{R}\right),
\end{array}
$$

diffusion to the left

$$
\emptyset_{i} 1_{i+1} \rightarrow 1_{i} \emptyset_{i+s_{1}}, \quad\left(\text { rate } \Gamma_{L}\right)
$$

\section{r$$
\emptyset_{i} 2_{i+1} \rightarrow 2_{i} \emptyset_{i+s_{2}}, \quad\left(\text { rate } \Gamma_{L}\right)
$$

and interchange of particles

$$
\begin{array}{ll}
1_{i} 2_{i+s_{1}} \rightarrow 2_{i} 1_{i+s_{2}}, & \left(\text { rate } \Gamma_{R}\right) \\
2_{i} 1_{i+s_{2}} \rightarrow 1_{i} 2_{i+s_{1}}, & \left(\text { rate } \Gamma_{L}\right) .
\end{array}
$$

As we see from (4), particles in the first class interchange positions with those of the second class with the same rate as they interchange positions with empty sites (diffusion). We should remark however that unless 
the second class particles have unit size $\left(s_{2}=1\right)$, the net effect of the second class particles in those of the first class is distinct from the effect produced by the holes, since as the result of the exchange the first class of particles will move by $s_{2}$ lattice size units, accelerating its diffusion.

The master equation (1) can be written as a Schrödinger equation in Euclidean time (see Ref. [3] for general application for two body processes)

$$
\frac{\partial \mid P>}{\partial t}=-H \mid P>
$$

if we interpret $\mid P>\equiv P(\{\beta\}, t)$ as the associated wave function. If we represent $\beta_{i}$ as $\mid \beta>_{i}$ the vector $\left|\beta>_{1} \otimes\right| \beta>_{2} \otimes \cdots \otimes \mid \beta>_{N}$ will give us the associated Hilbert space. The process (2)-(4) gives us the Hamiltonian (see Ref. [3] for general applications)

$$
\begin{aligned}
H= & D \sum_{j} H_{j} \\
H_{j}= & -\mathcal{P}\left\{\sum_{\alpha=1}^{2}\left[\epsilon_{+}\left(E_{j}^{0 \alpha} E_{j+1}^{\alpha 0}-E_{j}^{\alpha \alpha} E_{j+1}^{00}\right)+\epsilon_{-}\left(E_{j}^{\alpha 0} E_{j+1}^{0 \alpha}-E_{j}^{00} E_{j+1}^{\alpha \alpha}\right)\right]\right. \\
& \left.+\epsilon_{+}\left(E_{j}^{21} E_{j+s_{2}}^{10} E_{j+s_{1}}^{02}-E_{j}^{11} E_{j+s_{2}}^{00} E_{j+s_{1}}^{22}\right)+\epsilon_{-}\left(E_{j}^{12} E_{j+s_{1}}^{20} E_{j+s_{2}}^{01}-E_{j}^{22} E_{j+s_{1}}^{00} E_{j+s_{2}}^{11}\right)\right\} \mathcal{P}
\end{aligned}
$$

with

$$
D=\Gamma_{R}+\Gamma_{L}, \quad \epsilon_{+}=\frac{\Gamma_{R}}{\Gamma_{R}+\Gamma_{L}}, \quad \epsilon_{-}=\frac{\Gamma_{L}}{\Gamma_{R}+\Gamma_{L}} \quad\left(\epsilon_{+}+\epsilon_{-}=1\right),
$$

and periodic boundary conditions. The matrices $E^{\alpha, \beta}$ are $3 \times 3$ matrices with a single nonzero element $\left(E^{\alpha, \beta}\right)_{i, j}=\delta_{\alpha, i} \delta_{\beta, j}(\alpha, \beta, i, j \in \mathrm{Z})$. The projector $\mathcal{P}$ in (6), projects out from the associated Hilbert space the vectors $\mid\{\beta\}>$ which represent forbidden positions of the molecules due to their finite size, which mathematically means that for all $i, j$ with $\beta_{i}, \beta_{j} \neq 0, \quad|i-j| \geq$ $s_{\beta_{i}}(j>i)$. The constant $\mathrm{D}$ in (6) fixes the time scale and for simplicity we chose $D=1$. A particular simplification of (6) occurs when molecules of the first and second class have the same size $s_{1}=s_{2}=s$. In this case the Hamiltonian can be expressed as an anisotropic nearest-neighbor interaction spin-1 $S U(3)$ chain. Moreover in the case where their sizes are unity $(s=1)$ the model may be related to the $S U(3)$ Sutherland model with twisted boundary conditions [17].

\section{The Bethe ansatz equations}

We present in this section the exact solution of the general quantum chain (6). Since the present paper is going to appear in a special issue where the main subject is nonequilibrium physics, we are going to present a pedagogical and self-contained derivation of the exact solution, that can easily be followed by a nonexpert in the arena of exactly solvable models in statistical mechanics.

Due to the conservation of particles in the diffusion and interchange processes the total numbers of particles $n_{1}$ and $n_{2}$ of particles of class 1 and 2 are good quantum numbers and consequently we can split the associated Hilbert space into block disjoint sectors labeled by the numbers $n_{1}$ and $n_{2}\left(n_{1}=0,1, \ldots, n ; n_{2}=n-n_{1} ; n=\right.$ $\left.n_{1}+n_{2}\right)$. We therefore consider the eigenvalue equation

$$
H\left|n_{1}, n_{2}>=E\right| n_{1}, n_{2}>\text {, }
$$

where

$$
\left|n_{1}, n_{2}>=\sum_{\{Q\}} \sum_{\{x\}} f\left(x_{1}, Q_{1} ; \ldots ; x_{n}, Q_{n}\right)\right| x_{1}, Q_{1} ; \ldots ; x_{n}, Q_{n}>.
$$


Here $\mid x_{1}, Q_{1} ; \ldots ; x_{n}, Q_{n}>$ means the configuration where a particle of class $Q_{i}\left(Q_{i}=1,2\right)$ is at position $x_{i}$ $\left(x_{i}=1, \ldots, N\right)$. The summation $\{Q\}=\left\{Q_{1}, \ldots, Q_{n}\right\}$ extends over all permutations of $n$ numbers in which $n_{1}$ terms are 1 and $n_{2}$ terms are 2 , while the summation $\{x\}=\left\{x_{1}, \ldots, x_{n}\right\}$ runs, for each permutation $\{Q\}$, in the set of the $n$ nondecreasing integers satisfying

$$
x_{i+1} \geq x_{i}+s_{Q_{i}}, \quad i=1, \ldots, n-1, \quad s_{Q_{1}} \leq x_{n}-x_{1} \leq N-s_{Q_{n}} .
$$

Before getting the results for general values of $n$ let us consider initially the cases where we have 1 or 2 particles.

$\mathbf{n}=\mathbf{1}$. For one particle on the chain (class 1 or 2 ), as a consequence of the translational invariance of (6) it is simple to verify directly that the eigenfunctions are the momentum- $k$ eigenfunctions

$$
\left|1,0>=\sum_{x=1}^{N} f(x, 1)\right| x, 1>, \quad \text { or } \quad\left|0,1>=\sum_{x=1}^{N} f(x, 2)\right| x, 2>,
$$

with

$$
f(x, 1)=f(x, 2)=e^{i k x}, \quad k=\frac{2 \pi l}{N}, l=0,1, \ldots, N-1
$$

and energy given by

$$
E=e(k) \equiv-\left(\epsilon_{-} e^{i k}+\epsilon_{+} e^{-i k}-1\right)
$$

$\mathbf{n}=2$. For two particles of classes $Q_{1}$ and $Q_{2}\left(Q_{1}, Q_{2}=1,2\right)$ on the lattice, the eigenvalue equation (8) gives us two distinct relations depending on the relative location of the particles. The first relation applies to the case in which a particle of class $Q_{1}\left(\right.$ size $\left.s_{Q_{1}}\right)$ is at position $x_{1}$ and a particle $Q_{2}$ (size $s_{Q_{2}}$ ) is at position $x_{2}$, where $x_{2}>x_{1}+s_{Q_{1}}$. We obtain in this case the relation

$$
\begin{aligned}
E f\left(x_{1}, Q_{1} ; x_{2}, Q_{2}\right)= & -\epsilon_{+} f\left(x_{1}-1, Q_{1} ; x_{2}, Q_{2}\right)-\epsilon_{-} f\left(x_{1}, Q_{1} ; x_{2}+1, Q_{2}\right) \\
& -\epsilon_{-} f\left(x_{1}+1, Q_{1} ; x_{2}, Q_{2}\right)-\epsilon_{+} f\left(x_{1}, Q_{1} ; x_{2}-1, Q_{2}\right) \\
& +2 f\left(x_{1}, Q_{1} ; x_{2}, Q_{2}\right),
\end{aligned}
$$

where we have used the relation $\epsilon_{+}+\epsilon_{-}=1$. This last equation can be solved promptly by the ansatz

$$
f\left(x_{1}, Q_{1} ; x_{2}, Q_{2}\right)=e^{i k_{1} x_{1}} e^{i k_{2} x_{2}}
$$

with energy

$$
E=e\left(k_{1}\right)+e\left(k_{2}\right) \text {. }
$$

Since this relation is symmetric under the interchange of $k_{1}$ and $k_{2}$, we can write a more general solution of (14) as

$$
\begin{aligned}
f\left(x_{1}, Q_{1} ; x_{2}, Q_{2}\right) & =\sum_{P} A_{P_{1}, P_{2}}^{Q_{1}, Q_{2}} e^{i\left(k_{P_{1}} x_{1}+k_{P_{2}} x_{2}\right)} \\
& =A_{1,2}^{Q_{1}, Q_{2}} e^{i\left(k_{1} x_{1}+k_{2} x_{2}\right)}+A_{2,1}^{Q_{1}, Q_{2}} e^{i\left(k_{2} x_{1}+k_{1} x_{2}\right)}
\end{aligned}
$$

with the same energy as in (16). In (17) the summation is over the permutations $P=P_{1}, P_{2}$ of $(1,2)$. The second relation applies when $x_{2}=x_{1}+s_{Q_{1}}$. In this case instead of (14) we have

$$
\begin{aligned}
& E f\left(x_{1}, Q_{1} ; x_{1}+s_{Q_{1}}, Q_{2}\right)=-\epsilon_{+} f\left(x_{1}-1, Q_{1} ; x_{1}+s_{Q_{2}}, Q_{2}\right)-\epsilon_{-} f\left(x_{1}, Q_{1} ; x_{1}+s_{Q_{1}}+1, Q_{2}\right) \\
& -\tilde{\epsilon}_{Q_{1}, Q_{2}} f\left(x_{1}, Q_{2} ; x_{1}+s_{Q_{2}}, Q_{1}\right)+\left(1+\tilde{\epsilon}_{Q_{1}, Q_{2}}\right) f\left(x_{1}, Q_{1} ; x_{1}+s_{Q_{1}}, Q_{2}\right),
\end{aligned}
$$

where

$$
\tilde{\epsilon}_{1,1}=\tilde{\epsilon}_{2,2}=0, \quad \tilde{\epsilon}_{1,2}=\epsilon_{+} \quad \text { and } \quad \tilde{\epsilon}_{2,1}=\epsilon_{-} .
$$

If we now substitute the ansatz (17) with the energy (16), the constants $A_{12}^{Q_{1}, Q_{2}}$ and $A_{21}^{Q_{1}, Q_{2}}$, initially arbitrary, should now satisfy

$$
\sum_{P}\left\{\left[\mathcal{D}_{P_{1}, P_{2}}+e^{i k_{P_{2}}}\left(1-\tilde{\epsilon}_{Q_{1}, Q_{2}}\right)\right] e^{i k_{P_{2}}\left(s_{Q_{1}}-1\right)} A_{P_{1}, P_{2}}^{Q_{1}, Q_{2}}+\tilde{\epsilon}_{Q_{1}, Q_{2}} e^{i k_{P_{2}} s_{Q_{2}}} A_{P_{1}, P_{2}}^{Q_{2}, Q_{1}}\right\}=0
$$


where

$$
\mathcal{D}_{i, j}=-\left(\epsilon_{+}+\epsilon_{-} e^{i\left(k_{i}+k_{j}\right)}\right) .
$$

At this point it is convenient to consider separately the case where $Q_{1}=Q_{2}$ from those where $Q_{1} \neq Q_{2}$. If $Q_{1}=Q_{2}=Q(Q=1,2)$ eq. (20) gives

$$
\sum_{P}\left(\mathcal{D}_{P_{1}, P_{2}}+e^{i k_{P_{2}}}\right) e^{i k_{P_{2}}\left(s_{Q}-1\right)} A_{P_{1}, P_{2}}^{Q_{1}, Q_{2}}=0
$$

and the cases $Q_{1} \neq Q_{2}$ give us the equations

$$
\sum_{P}\left[\begin{array}{cc}
\mathcal{D}_{P_{1}, P_{2}}+e^{i k_{P_{2}}} \epsilon_{-} & \epsilon_{+} e^{i k_{P_{2}}} \\
\epsilon_{-} e^{i k_{P_{2}}} & \mathcal{D}_{P_{1}, P_{2}}+\epsilon_{+} e^{i k_{P_{2}}}
\end{array}\right]\left[\begin{array}{c}
e^{i k_{P_{2}}\left(s_{1}-1\right)} A_{P_{1}, P_{2}}^{1,2} \\
e^{i k_{P_{2}}\left(s_{2}-1\right)} A_{P_{1}, P_{2}}^{2,1}
\end{array}\right]=0 .
$$

Performing the above summation we obtain, after lengthy but straightforward algebra, the following relation among the amplitudes

$$
\left[\begin{array}{c}
A_{1,2}^{1,2} e^{i k_{2}\left(s_{1}-1\right)} \\
A_{1,2}^{2,1} e^{i k_{2}\left(s_{2}-1\right)}
\end{array}\right]=-\frac{\mathcal{D}_{1,2}+e^{i k_{1}}}{\mathcal{D}_{1,2}+e^{i k_{2}}}\left\{1-\Phi\left(k_{1}, k_{2}\right)\left[\begin{array}{cc}
\epsilon_{+} & -\epsilon_{+} \\
-\epsilon_{-} & \epsilon_{-}
\end{array}\right]\right\}\left[\begin{array}{c}
A_{2,1}^{12} e^{i k_{1}\left(s_{1}-1\right)} \\
A_{2,1}^{21} e^{i k_{1}\left(s_{2}-1\right)}
\end{array}\right]
$$

where

$$
\Phi\left(k_{1}, k_{2}\right)=\frac{e^{i k_{1}}-e^{i k_{2}}}{\mathcal{D}_{1,2}+e^{i k_{1}}} .
$$

Equations (22) and (23) can be written in a compact form

$$
A_{P_{1}, P_{2}}^{Q 1, Q 2}=-\Xi_{P_{1}, P_{2}} \sum_{Q_{1}^{\prime}, Q_{2}^{\prime}=1}^{2} S_{Q_{1}^{\prime}, Q_{2}^{\prime}}^{Q_{1}, Q_{2}}\left(k_{P_{1}}, k_{P_{2}}\right) A_{P_{2}, P_{1}}^{Q_{2}^{\prime}, Q_{1}^{\prime}}, \quad\left(Q_{1}, Q_{2}=1,2\right)
$$

with

$$
\Xi_{l, j}=\frac{\mathcal{D}_{l, j}+e^{i k_{l}}}{\mathcal{D}_{l, j}+e^{i k_{j}}}=\frac{\epsilon_{+}+\epsilon_{-} e^{i\left(k_{l}+k_{j}\right)}-e^{i k_{l}}}{\epsilon_{+}+\epsilon_{-} e^{i\left(k_{l}+k_{j}\right)}-e^{i k_{j}}},
$$

where we have introduced the $S$ matrix. From 22 and (23), the only non zero elements of $S_{Q_{1}^{\prime}, Q_{2}^{\prime}}^{Q_{1}, Q_{2}}$ are:

$$
\begin{aligned}
& S_{1,1}^{1,1}\left(k_{1}, k_{2}\right)=e^{i\left(k_{1}-k_{2}\right)\left(s_{1}-1\right)}, \quad s_{2,2}^{2,2}\left(k_{1}, k_{2}\right)=e^{i\left(k_{1}-k_{2}\right)\left(s_{2}-1\right)} \\
& S_{2,1}^{1,2}\left(k_{1}, k_{2}\right)=\left[1-\epsilon_{+} \Phi\left(k_{1}, k_{2}\right)\right] e^{i\left(k_{1}-k_{2}\right)\left(s_{1}-1\right)} \\
& S_{1,2}^{2,1}\left(k_{1}, k_{2}\right)=\left[1-\epsilon_{-} \Phi\left(k_{1}, k_{2}\right)\right] e^{i\left(k_{1}-k_{2}\right)\left(s_{2}-1\right)} \\
& S_{1,2}^{1,2}\left(k_{1}, k_{2}\right)=\epsilon_{+} \Phi\left(k_{1}, k_{2}\right) e^{i k_{1}\left(s_{2}-1\right)} e^{-i k_{2}\left(s_{1}-1\right)} \\
& S_{2,1}^{2,1}\left(k_{1}, k_{2}\right)=\epsilon_{-} \Phi\left(k_{1}, k_{2}\right) e^{i k_{1}\left(s_{1}-1\right)} e^{-i k_{2}\left(s_{2}-1\right)}
\end{aligned}
$$

A graphical representation of the S matrix is shown in Fig. 2a. Equations (24) do not fix the "wave numbers" $k_{1}$ and $k_{2}$. In general, these numbers are complex, and are fixed due to the cyclic boundary condition

$$
f\left(x_{1}, Q_{1} ; x_{2}, Q_{2}\right)=f\left(x_{2}, Q_{2} ; x_{1}+N, Q_{1}\right),
$$

which from (17) gives the relation

$$
A_{1,2}^{Q_{1} Q_{2}}=e^{i k_{1} N} A_{2,1}^{Q_{2}, Q_{1}}, \quad A_{2,1}^{Q_{1}, Q_{2}}=e^{i k_{2} N} A_{2,1}^{Q_{2}, Q_{1}} .
$$

This last equation, when solved by exploiting (24)-(26), gives us the possible values of $k_{1}$ and $k_{2}$, and from (16) the eigenenergies in the sector with 2 particles. Instead of solving these equations for the particular case $n=2$ let us now consider the case of general $n$. 
(a)

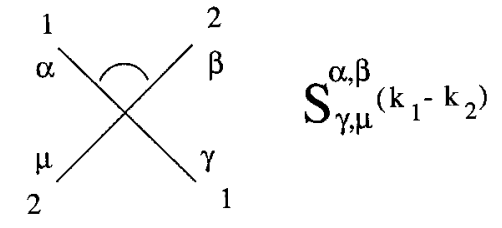

2

(b)

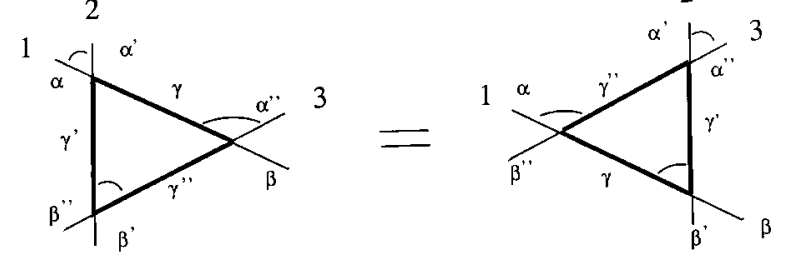

Figure 2. Graphical representations of: a) the $S$ matrix (26) and b) the Yang-Baxter equations (34).

General $\mathbf{n}$. The above calculation can be generalized for arbitrary values of $n_{1}$ and $n_{2}$ of particles of classes 1 and 2 , respectively $\left(n_{1}+n_{2}=n\right)$. The ansatz for the wave function (9) becomes

$$
f\left(x_{1}, Q_{1} ; \ldots ; x_{n}, Q_{n}\right)=\sum_{P} A_{P_{1}, \ldots, P_{n}}^{Q_{1}, \ldots, Q_{n}} e^{i\left(k_{P_{1}} x_{1}+\cdots+k_{P_{n}} x_{n}\right)}
$$

where the sum extends over all permutations $P$ of the integers $1,2, \ldots, n$. For the components $\left|x_{1}, Q_{1} ; \ldots ; x_{n}, Q_{n}\right\rangle$ where $x_{i+1}-x_{i}>s_{Q_{i}}$ for $i=1,2, \ldots, n$, it is simple to see that the eigenvalue equation ( 8 ) is satisfied by the ansatz (29) with energy

$$
E=\sum_{j=1}^{n} e\left(k_{j}\right)
$$

On the other hand if a pair of particles of class $Q_{i}, Q_{i+1}$ is at positions $x_{i}, x_{i+1}$, where $x_{i+1}=x_{i}+s_{Q_{i}}$, equation (8) with the ansatz (29) and the relation (30) give us the generalization of relation (24), namely

$$
A_{\ldots, P_{i}, P_{i+1}, \ldots}^{\cdots, Q_{i}, Q_{i+1}, \cdots}=-\Xi_{P_{i}, P_{i+1}} \sum_{Q_{1}^{\prime}, Q_{2}^{\prime}}^{2} S_{Q_{1}^{\prime}, Q_{2}^{\prime}}^{Q_{i}, Q_{i+1}}\left(k_{P_{i}}, k_{P_{i+1}}\right) A_{\ldots, P_{i+1}, P_{i}, \ldots}^{\cdots, Q_{2}^{\prime}, Q_{1}^{\prime}, \ldots} \quad Q_{i}, Q_{i+1}=1,2,
$$

with $S$ given by eq. (26). Inserting the ansatz (29) in the boundary condition

$$
f\left(x_{1}, Q_{1} ; \ldots ; x_{n}, Q_{n}\right)=f\left(x_{2}, Q_{2} ; \ldots ; x_{n}, Q_{n} ; x_{1}+N, Q_{1}\right)
$$

we obtain the additional relation

$$
A_{P_{1}, \ldots, P_{n}}^{Q_{1}, \cdots, Q_{n}}=e^{i k_{P_{1}} N} A_{P_{2}, \ldots, P_{n}, P_{1}}^{Q_{2}, \cdots, Q_{n}, Q_{1}}
$$

which together with (31) should give us the energies.

Successive applications of (31) give us in general distinct relations between the amplitudes. For example $A_{\ldots, k_{1}, k_{2}, k_{3}, \ldots}^{\ldots, \alpha, \beta, \gamma}$ relate to $A_{\ldots, k_{3}, k_{2}, k_{1}, \ldots}^{\ldots, \gamma, \alpha, \ldots}$ by performing the permutations $\alpha \beta \gamma \rightarrow \beta \alpha \gamma \rightarrow \beta \gamma \alpha \rightarrow \gamma \beta \alpha$ or $\alpha \beta \gamma \rightarrow$ $\alpha \gamma \beta \rightarrow \gamma \alpha \beta \rightarrow \gamma \beta \alpha$, and consequently the $S$-matrix should satisfy the Yang-Baxter [16, 23] equation

$$
\begin{gathered}
\sum_{\gamma, \gamma^{\prime}, \gamma^{\prime \prime}=1}^{2} S_{\gamma, \gamma^{\prime}}^{\alpha, \alpha^{\prime}}\left(k_{1}-k_{2}\right) S_{\beta, \gamma^{\prime \prime}}^{\gamma, \alpha^{\prime \prime}}\left(k_{1}-k_{3}\right) S_{\beta^{\prime}, \beta^{\prime \prime}}^{\gamma^{\prime}, \gamma^{\prime \prime}}\left(k_{2}-k_{3}\right)= \\
\sum_{\gamma, \gamma^{\prime}, \gamma^{\prime \prime}=1}^{2} S_{\gamma^{\prime}, \gamma^{\prime \prime}}^{\alpha^{\prime}, \alpha^{\prime \prime}}\left(k_{2}-k_{3}\right) S_{\gamma, \beta^{\prime \prime}}^{\alpha, \gamma^{\prime \prime}}\left(k_{1}-k_{3}\right) S_{\beta, \beta^{\prime}}^{\gamma, \gamma^{\prime}}\left(k_{1}-k_{2}\right),
\end{gathered}
$$

for $\alpha, \alpha^{\prime}, \alpha^{\prime \prime}, \beta, \beta^{\prime}, \beta^{\prime \prime}=1,2$ and $S$ given by (26). In Fig. $2 \mathrm{~b}$ we show graphically this equation. Actually the relation (34) is a necessary and sufficient condition [16, 23] to obtain a non-trivial solution for the amplitudes in Eq. (31).

We can verify by a long and straightforward calculation that for arbitrary values of $s_{1}$ and $s_{2}$, the $S$ matrix (26), satisfies the Yang-Baxter equation (34), and consequently we may use relations (31) and (33) to obtain the eigenenergies of the Hamiltonian (6). Applying relation (31) $n$ times on the right of equation (33) we obtain a 
relation between the amplitudes with the same momenta. Using the graphical representation in Fig. 2a for the $S$ matrix, we illustrate in Fig. 4 the result of such applications. We then obtain

$$
\begin{array}{r}
A_{P_{1}, \ldots, P_{n}}^{Q_{1}, \ldots, Q_{n}}=e^{i k_{P_{1}} N} A_{P_{2}, \ldots, P_{n}, P_{1}}^{Q_{2}, \ldots, Q_{n}, Q_{1}}=\left(\prod_{i=2}^{n}-\Xi_{P_{i}, P_{1}}\right) e^{i k_{P_{1}} N} \sum_{Q_{1}^{\prime}, \ldots, Q_{n}^{\prime} Q_{1}^{\prime \prime}, \ldots, Q_{n}^{\prime \prime}} \sum_{S_{Q_{1}^{\prime}, Q_{1}^{\prime \prime}}^{Q_{1}, Q_{2}^{\prime \prime}}\left(k_{P_{1}}, k_{P_{1}}\right) S_{Q_{2}^{\prime}, Q_{2}^{\prime \prime}}^{Q_{2}, Q_{3}^{\prime \prime}}\left(k_{P_{2}}, k_{P_{1}}\right) \cdots S_{Q_{n-1}^{\prime}, Q_{n-1}^{\prime \prime}}^{Q_{n-1}, Q_{n}^{\prime \prime}}\left(k_{P_{n-1}}, k_{P_{1}}\right) S_{Q_{n}^{\prime}, Q_{n}^{\prime \prime}}^{Q_{n}, Q_{1}^{\prime \prime}}\left(k_{P_{n}}, k_{P_{1}}\right) A_{P_{1}, \ldots, P_{n}}^{Q_{1}^{\prime}, \ldots, Q_{n}^{\prime}},}
\end{array}
$$

where we have introduced the harmless extra sum

$$
1=\sum_{Q_{1}^{\prime \prime}, Q_{2}^{\prime \prime}=1}^{2} \delta_{Q_{2}^{\prime \prime}, Q_{1}^{\prime}} \delta_{Q_{1}^{\prime \prime}, Q_{1}}=\sum_{Q_{1}^{\prime \prime}, Q_{2}^{\prime \prime}=1}^{2} S_{Q_{1}^{\prime}, Q_{1}^{\prime \prime}}^{Q_{1}, Q^{\prime \prime}}\left(k_{P_{1}}-k_{P_{1}}\right) .
$$

In order to fix the values of $\left\{k_{j}\right\}$ we should solve (35), i.e., we should find the eigenvalues $\Lambda(k)$ of the matrix

$$
T(k)_{\left\{Q^{\prime}\right\}}^{\{Q\}}=\sum_{Q_{1}^{\prime \prime}, \ldots, Q_{n}^{\prime \prime}=1}^{2}\left\{\left(\prod_{l=1}^{n-1} S_{Q_{l}^{\prime}, Q_{l}^{\prime \prime}}^{Q_{l}, Q_{\prime+1}^{\prime \prime}}\left(k_{P_{l}}-k\right)\right) S_{Q_{n}^{\prime}, Q_{n}^{\prime \prime}}^{Q_{n}, Q^{\prime \prime}}\left(k_{P_{n}}, k\right)\right\}
$$

and the Bethe-ansatz equations which fix the set $\left\{k_{l}\right\}$ will be given from (35) by

$$
e^{-i k_{j} N}=(-1)^{n-1}\left(\prod_{l=1}^{n} \Xi_{l, j}\right) \Lambda\left(k_{j}\right), \quad j=1, \ldots, n
$$

Using the graphical representation for $S$ given in Fig. 2a the matrix $T$ in (37) can be represented graphically as in Fig. 5, and we identify $T(k)$ as the transfer matrix of an inhomogeneous 6-vertex model, on a periodic lattice, with Boltzmann weights $S_{Q_{1}^{\prime}, Q_{2}^{\prime}}^{Q_{1}, Q_{2}}\left(k_{P_{l}}, k\right)(l=1, \ldots, n)$. Consequently, in order to obtain the eigenenergies of the quantum chain (6) we should diagonalize the above transfer matrix $T(k)$.
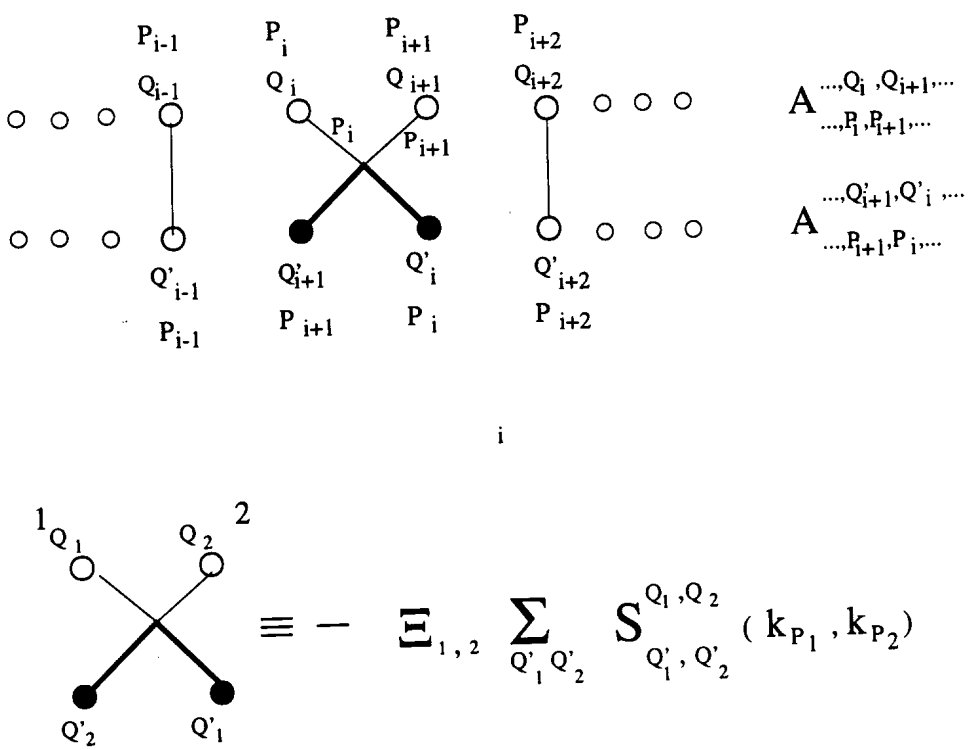

Figure 3. Graphical representation of the relation (31). The circles represents the particle's indices $\{Q\}$ and $\left\{Q^{\prime}\right\}$, and the lines the "wave numbers" $\left\{k_{P_{i}}\right\}$. The variables represented in the full circles are summed. 


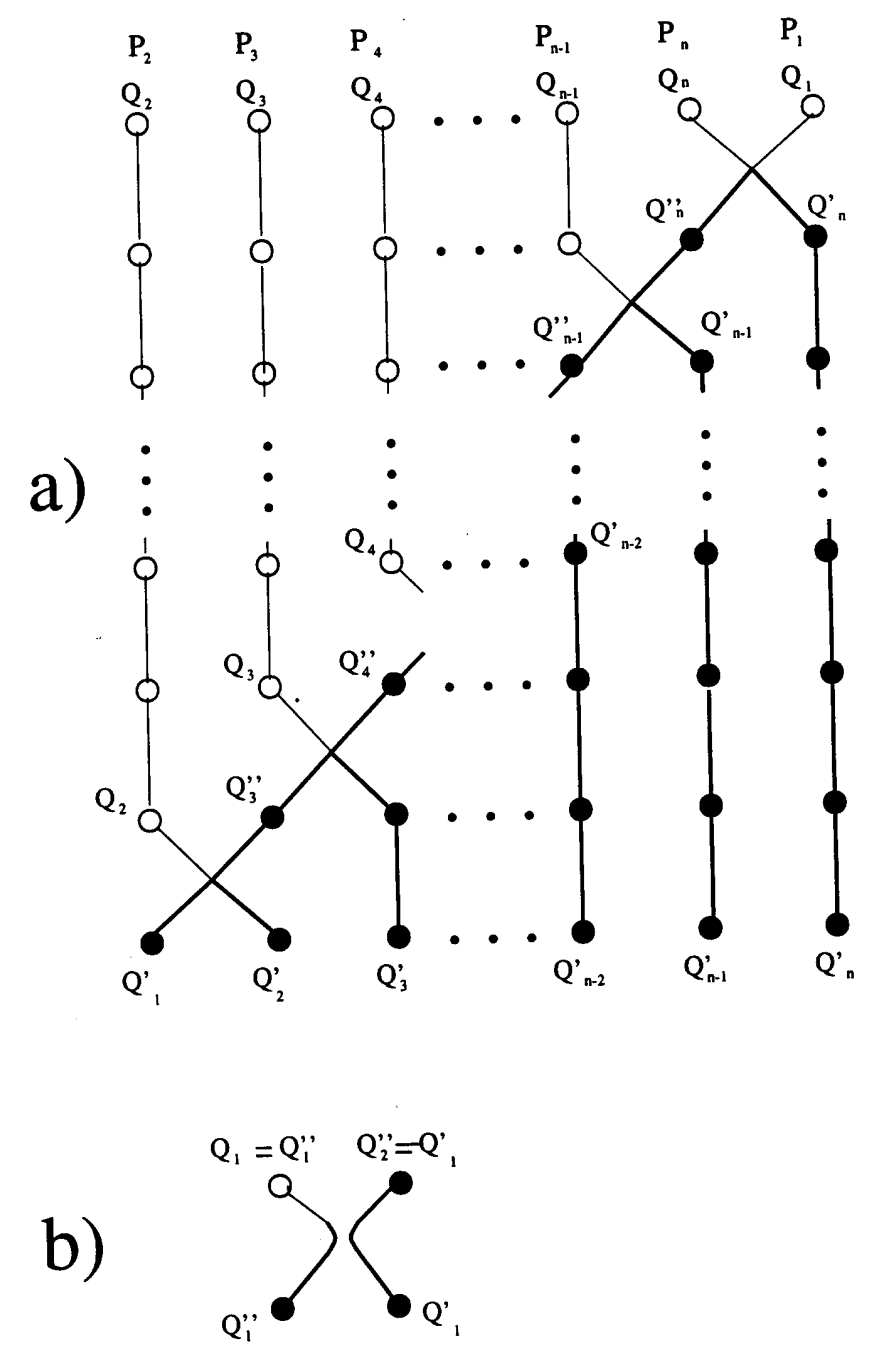

Figure 4. a) Graphical representation of the result of $n$ iterations of relation (31) on the right of equation (33). The diagramatic representation of Fig. 3 was used. b) A graphical representation of equation (36).

\section{Diagonalization of $T(k)$}

The simplest way to diagonalize $T$ is through the introduction of the monodromy matrix $\mathcal{M}(k)[21]$, which is a transfer matrix of the inhomogeneous vertex model under consideration, where, instead of being periodic, the first and last link in the horizontal direction are fixed to the values $\mu_{1}$ and $\mu_{n+1}\left(\mu_{1}, \mu_{n+1}=1,2\right)$, that is

$$
\mathcal{M}_{\left\{Q^{\prime}\right\}, \mu_{1}}^{\{Q\}, \mu_{n+1}}(k)=\sum_{\mu_{2}, \ldots, \mu_{n}} S_{Q_{1}^{\prime}, \mu_{1}}^{Q_{1}, \mu_{2}}\left(k_{P_{1}}, k\right) S_{Q_{2}^{\prime}, \mu_{2}}^{Q_{2}, \mu_{3}}\left(k_{P_{2}}, k\right) \cdots S_{Q_{n-1}^{\prime}, \mu_{n-1}}^{Q_{n-1}, \mu_{n}}\left(k_{P_{n-1}}, k\right) S_{Q_{n}^{\prime}, \mu_{n}}^{Q_{n}, \mu_{n+1}}\left(k_{P_{n}}, k\right) .
$$

The monodromy matrix $\mathcal{M}_{\left\{Q^{\prime}\right\}, \mu_{1}}^{\{Q\}, \mu_{n+1}}(k)$ is represented in Fig. 6a and has coordinates $\{Q\},\left\{Q^{\prime}\right\}$ in the vertical space ( $2^{n}$ dimensions) and coordinates $\mu_{1}, \mu_{n+1}$ in the horizontal space (4 dimensions). This matrix satisfies the following important relations

$$
S_{\nu_{1}, \mu_{1}}^{\nu_{1}^{\prime}, \mu_{1}^{\prime}}\left(k^{\prime}, k\right) \mathcal{M}_{\left\{\alpha_{l}\right\}, \mu_{1}^{\prime}}^{\left\{\gamma_{l}\right\}, \mu_{n+1}}(k) \mathcal{M}_{\left\{\gamma_{l}\right\}, \nu_{1}^{\prime}}^{\left\{\beta_{l}\right\}, \nu_{n+1}}\left(k^{\prime}\right)=\mathcal{M}_{\left\{\alpha_{l}\right\}, \nu_{1}}^{\left\{\gamma_{l}\right\}, \nu_{n+1}^{\prime}}\left(k^{\prime}\right) \mathcal{M}_{\left\{\gamma_{l}\right\}, \mu_{1}}^{\left\{\beta_{l}\right\}, \mu_{n+1}^{\prime}}(k) S_{\nu_{n+1}^{\prime}, \mu_{n+1}^{\prime}}^{\nu_{n+1}, \mu_{n+1}}\left(k^{\prime}, k\right) .
$$

This relation is shown graphically in Fig. 6b; its validity follows directly from successive applications of the YangBaxter equations (34) (see Fig. 2b). by

In order to exploit relation (40) let us denote the components of the monodromy matrix in the horizontal space

$$
\begin{aligned}
& A(k)=A(k)_{\left\{\alpha_{l}\right\}}^{\left\{\gamma_{l}\right\}}=\mathcal{M}_{\left\{\alpha_{l}\right\}, 1}^{\left\{\gamma_{l}\right\}, 1}(k), \quad B(k)=B(k)_{\left\{\alpha_{l}\right\}}^{\left\{\gamma_{l}\right\}}=\mathcal{M}_{\left\{\alpha_{l}\right\}, 1}^{\left\{\gamma_{l}\right\}, 2}(k), \\
& C(k)=C(k)_{\left\{\alpha_{l}\right\}}^{\left\{\gamma_{l}\right\}}=\mathcal{M}_{\left\{\alpha_{l}\right\}, 2}^{\left\{\gamma_{l}\right\}, 1}(k), \quad D(k)=D(k)_{\left\{\alpha_{l}\right\}}^{\left\{\gamma_{l}\right\}}=\mathcal{M}_{\left\{\alpha_{l}\right\}, 2}^{\left\{\gamma_{l}\right\}, 2}(k),
\end{aligned}
$$


and clearly the transfer matrix $T(k)$ of the periodic inhomogeneous lattice, we want to diagonalize, is given by

$$
T(k)=A(k)+D(k) \text {. }
$$

As a consequence of (40) the matrices $A, B, C$ and $D$ in (41) obey some algebraic relations. The elements $\left(\nu_{1}, \mu_{1}, \nu_{n+1}, \mu_{n+1}\right)=(1,1,2,2),(1,1,1,2)$ and $(1,2,2,2)$ give us the relations

$$
\begin{gathered}
{\left[B(k), B\left(k^{\prime}\right)\right]=0,} \\
A(k) B\left(k^{\prime}\right)=\frac{S_{1,1}^{1,1}\left(k, k^{\prime}\right)}{S_{1,2}^{1,2}\left(k, k^{\prime}\right)} B\left(k^{\prime}\right) A(k)-\frac{S_{2,1}^{1,2}\left(k, k^{\prime}\right)}{S_{1,2}^{1,2}\left(k, k^{\prime}\right)} B(k) A\left(k^{\prime}\right), \\
D(k) B\left(k^{\prime}\right)=\frac{S_{2,2}^{2,2}\left(k^{\prime}, k\right)}{S_{1,2}^{1,2}\left(k^{\prime}, k\right)} B\left(k^{\prime}\right) D(k)-\frac{S_{1,2}^{2,1}\left(k^{\prime}, k\right)}{S_{1,2}^{1,2}\left(k^{\prime}, k\right)} B(k) D\left(k^{\prime}\right),
\end{gathered}
$$

respectively. The diagonalization of $T(k)$ in $(42)$ will be done by exploiting the above relations. This procedure is known in the literature as the algebraic Bethe ansatz [21]. The first step in this method follows from the identification of a reference state $\mid \Omega>$, which should be an eigenstate of $A(k)$ and $D(k)$, and hence $T(k)$, but not of $B(k)$. In our problem it is simple to see that a possible reference state is $|\Omega>=|\left\{\alpha_{l}=1\right\}>_{l=1, \ldots, n}$, which corresponds to a state with first-class particles only. It is simple to calculate

$$
\begin{aligned}
& A(k)|\Omega>=a(k)| \Omega>, \quad D(k)|\Omega>=d(k)| \Omega>, \\
& C(k)|\Omega>=0, \quad B(k)| \Omega>=\sum_{i=1}^{n} b_{i}(k) \mid \Omega>,
\end{aligned}
$$

where

$$
\begin{aligned}
a(k) & =\prod_{i=1}^{n} S_{1,1}^{1,1}\left(k_{P_{i}}, k\right), \quad d(k)=\prod_{i=1}^{n} S_{2,1}^{1,2}\left(k_{P_{i}}, k\right), \\
b_{i}(k) & =\prod_{l=1}^{i-1} S_{1,1}^{1,1}\left(k_{P_{l}}, k\right) \prod_{l=j}^{n} S_{1,2}^{1,2}\left(k_{P_{l}}, k\right) .
\end{aligned}
$$

In Fig. 7 we show a graphical representation of the above relation. The idea in the algebraic Bethe ansatz is that $B(k)$ acts as a creation operator in the reference ("vacuum") state, i.e., it creates particles of second class in a sea of particles of first class $\mid \Omega>$, and it is expected that a general eigenvector of $T(k)$ in the sector with $n_{2}$ second-class particles will be given by the ansatz

$$
\Psi\left(k_{1}^{(1)}, k_{2}^{(1)}, \ldots, k_{n_{2}}^{(1)}\right)=B\left(k_{1}^{(1)}\right) B\left(k_{2}^{(1)}\right) \cdots B\left(k_{n_{2}}^{(1)}\right) \mid \Omega>.
$$

The numbers $\left\{k_{i}^{(1)}\right\}, i=1, \ldots, n_{2}$ here play the role of the "wave number" $\left\{k_{i}\right\}$ in the coordinate Bethe ansatz (29), and are going to be fixed by the eigenvalue equation

$$
T(k) \Psi\left(k_{1}^{(1)}, \ldots, k_{n_{2}}^{(1)}\right)=(A(k)+D(k)) \Psi\left(k_{1}^{(1)}, \ldots, k_{n_{2}}^{(1)}\right)=\Lambda(k) \Psi\left(k_{1}^{(1)}, \ldots, k_{n_{2}}^{(1)}\right) .
$$

Before deriving the relation for general values of $n_{2}$, let us consider initially the cases where $n_{2}=1$ and $n_{2}=2$.

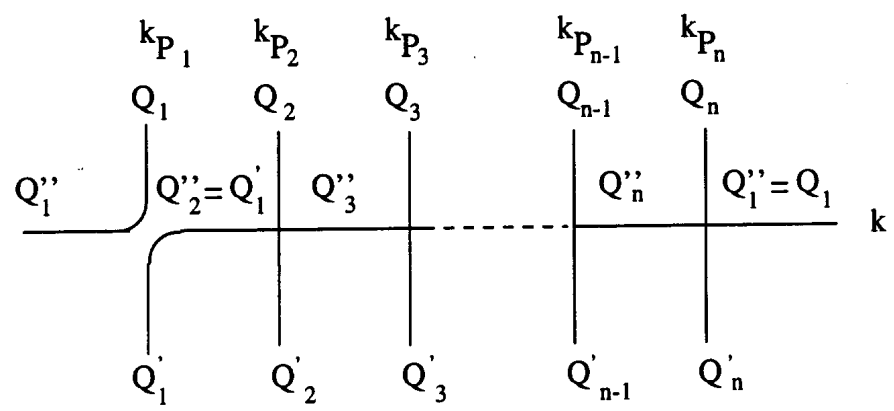

Figure 5. Graphical representation of the transfer matrix $T(k)$, of the inhomogeneous 6-vertex model, in a periodic lattice of size $n$. 


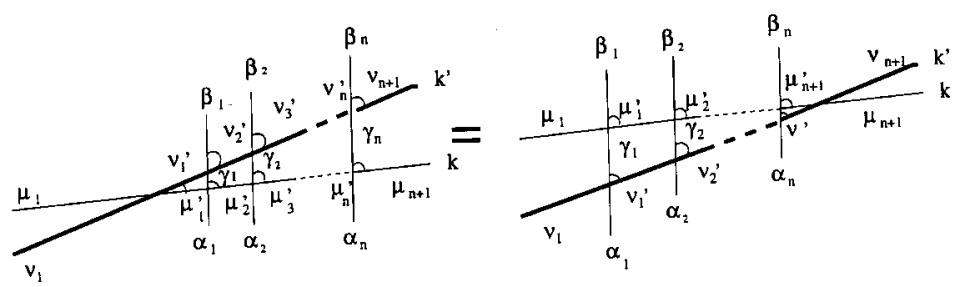

Figure 6. Graphical representation of the relation (40) between the $S$ matrix and the monodromy matrix $\mathcal{M}$.

$\mathbf{n}_{\mathbf{2}}=\mathbf{1}$. Using relation (44) and (45) in (49) we obtain

$$
\begin{aligned}
T(k) \Psi\left(k_{1}^{(1)}\right) & =\left[\frac{S_{1,1}^{1,1}\left(k, k_{1}^{(1)}\right)}{S_{1,2}^{1,2}\left(k, k_{1}^{(1)}\right)}+d(k) \frac{S_{2,2}^{2,2}\left(k_{1}^{(1)}, k\right)}{S_{1,2}^{1,2}\left(k_{1}^{(1)}, k\right)}\right] \Psi\left(k_{1}^{(1)}\right) \\
& -\left[\frac{S_{2,1}^{1,2}\left(k, k_{1}^{(1)}\right)}{S_{1,2}^{1,2}\left(k, k_{1}^{(1)}\right)}+d\left(k_{1}^{(1)}\right) \frac{S_{1,2}^{2,1}\left(k_{1}^{(1)}, k\right)}{S_{1,2}^{1,2}\left(k_{1}^{(1)}, k\right)}\right] B(k) \mid \Omega>=\Lambda(k) \Psi\left(k_{1}^{(1)}\right),
\end{aligned}
$$

which is clearly satisfied if the coefficient of $B(k) \mid \Omega>$ (unwanted term) vanishes. This condition, which fixes $k_{1}^{(1)}$, is given by

$$
\prod_{j=1}^{n} S_{1,2}^{1,2}\left(k_{P_{j}}, k_{1}^{(1)}\right)=1
$$

where we used the relation

$$
\frac{S_{2,1}^{1,2}\left(k, k^{\prime}\right)}{S_{1,2}^{1,2}\left(k, k^{\prime}\right)}=-\frac{S_{1,2}^{2,1}\left(k^{\prime}, k\right)}{S_{1,2}^{1,2}\left(k^{\prime}, k\right)},
$$

valid for the $S$ matrix (26). The eigenvalue is given by

$$
\Lambda(k)=\frac{S_{1,1}^{1,1}\left(k, k_{1}^{(1)}\right)}{S_{1,2}^{1,2}\left(k, k_{1}^{(1)}\right)}+\frac{S_{2,2}^{2,2}\left(k_{1}^{(1)}, k\right)}{S_{1,2}^{1,2}\left(k_{1}^{(1)}, k\right)} \prod_{j=1}^{n} S_{1,2}^{1,2}\left(k_{P_{j}}, k\right),
$$

provided (51)is satisfied.

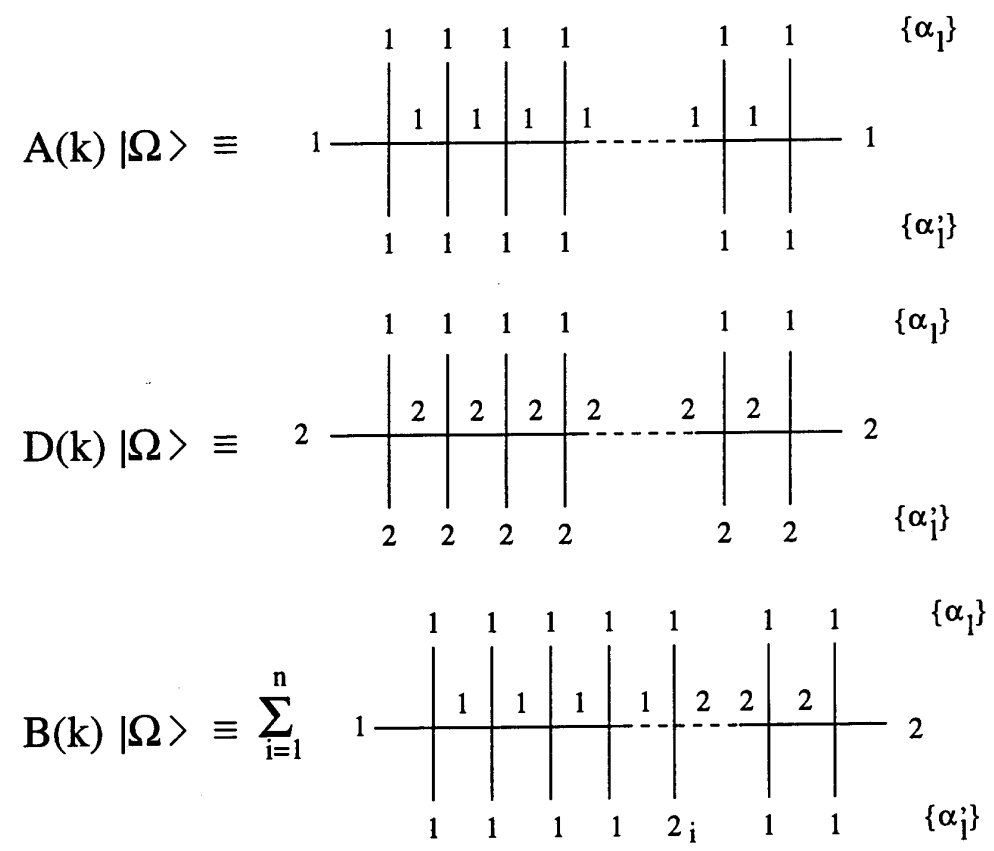

Figure 7. Graphical representation of the relations (46). 
$\mathbf{n}_{\mathbf{2}}=\mathbf{2}$. In this case the application of $A(k)$ and $D(k)$ in the ansatz (48) give us

$$
\begin{aligned}
A(k) \Psi\left(k_{1}^{(1)}, k_{2}^{(1)}\right) & =\frac{S_{1,1}^{1,1}\left(k, k_{1}^{(1)}\right) S_{1,1}^{1,1}\left(k, k_{2}^{(1)}\right)}{S_{1,2}^{1,2}\left(k, k_{1}^{(1)}\right) S_{1,2}^{1,2}\left(k, k_{2}^{(1)}\right)} a(k) \Psi\left(k_{1}^{(1)}, k_{2}^{(1)}\right) \\
& -\frac{S_{2,1}^{1,2}\left(k, k_{1}^{(1)}\right) S_{1,1}^{1,1}\left(k_{1}^{(1)}, k_{2}^{(1)}\right)}{S_{1,2}^{1,2}\left(k, k_{1}^{(1)}\right) S_{1,2}^{1,2}\left(k_{1}^{(1)}, k_{2}^{(1)}\right)} a\left(k_{1}^{(1)}\right) B(k) B\left(k_{2}^{(1)}\right) \mid \Omega> \\
& -\frac{S_{2,1}^{1,2}\left(k, k_{2}^{(1)}\right) S_{1,1}^{1,1}\left(k_{2}^{(1)}, k_{1}^{(1)}\right)}{S_{1,2}^{1,2}\left(k, k_{2}^{(1)}\right) S_{1,2}^{1,2}\left(k_{2}^{(1)}, k_{1}^{(1)}\right)} a\left(k_{2}^{(1)}\right) B(k) B\left(k_{1}^{(1)}\right) \mid \Omega>,
\end{aligned}
$$

and

$$
\begin{aligned}
D(k) \Psi\left(k_{1}^{(1)}, k_{2}^{(1)}\right) & =\frac{S_{2,2}^{2,2}\left(k_{1}^{(1)}, k\right) S_{2,2}^{2,2}\left(k_{2}^{(1)}, k\right)}{S_{1,2}^{1,2}\left(k_{1}^{(1)}, k\right) S_{1,2}^{1,2}\left(k_{2}^{(1)}, k\right)} d(k) \Psi\left(k_{1}^{(1)}, k_{2}^{(1)}\right) \\
& -\frac{S_{1,2}^{2,1}\left(k_{1}^{(1)}, k\right) S_{2,2}^{2,2}\left(k_{2}^{(1)}, k_{1}^{(1)}\right)}{S_{1,2}^{1,2}\left(k_{1}^{(1)}, k\right) S_{1,2}^{1,2}\left(k_{2}^{(1)}, k_{1}^{(1)}\right)} d\left(k_{1}^{(1)}\right) B(k) B\left(k_{2}^{(1)}\right) \mid \Omega> \\
& -\frac{S_{1,2}^{2,1}\left(k_{2}^{(1)}, k\right) S_{2,2}^{2,2}\left(k_{1}^{(1)}, k_{2}^{(1)}\right)}{S_{1,2}^{1,2}\left(k_{2}^{(1)}, k\right) S_{1,2}^{1,2}\left(k_{1}^{(1)}, k_{2}^{(1)}\right)} d\left(k_{2}^{(1)}\right) B(k) B\left(k_{1}^{(1)}\right) \mid \Omega>,
\end{aligned}
$$

where besides relations (44) and (45) we have used (43), which implies $\Psi\left(k_{1}^{(1)}, k_{2}^{(1)}\right)=\Psi\left(k_{2}^{(1)}, k_{1}^{(1)}\right)$. From (54) and (55) the condition (49) gives us

$$
\Lambda(k)=\prod_{i=1}^{n} S_{1,1}^{1,1}\left(k_{P_{i}}, k\right) \prod_{j=1}^{2} \frac{S_{1,1}^{1,1}\left(k, k_{j}^{(1)}\right)}{S_{1,2}^{1,2}\left(k, k_{j}^{(1)}\right)}+\prod_{i=1}^{n} S_{1,2}^{1,2}\left(k_{P_{i}}, k\right) \prod_{j=1}^{2} \frac{S_{2,2}^{2,2}\left(k_{j}^{(1)}, k\right)}{S_{1,2}^{1,2}\left(k_{j}^{(1)}, k\right)},
$$

under the condition, which fixes $k_{1}^{(1)}$ and $k_{2}^{(1)}$,

$$
\prod_{i=1}^{n} S_{1,2}^{1,2}\left(k_{P_{i}}, k_{\alpha}^{(1)}\right)=\prod_{\beta=1(\neq \alpha)}^{2} \frac{S_{1,1}^{1,1}\left(k_{\alpha}^{(1)}, k_{\beta}^{(1)}\right) S_{1,2}^{1,2}\left(k_{\beta}^{(1)}, k_{\alpha}^{(1)}\right)}{S_{1,2}^{1,2}\left(k_{\alpha}^{(1)}, k_{\beta}^{(1)}\right) S_{2,2}^{2,2}\left(k_{\beta}^{(1)}, k_{\alpha}^{(1)}\right)} \prod_{j=1}^{n} S_{1,1}^{1,1}\left(k_{P_{j}}, k_{\alpha}^{(1)}\right) \quad \alpha=1,2 .
$$

In deriving this last expression the relation (52) was also used.

General $\mathbf{n}_{\mathbf{2}}$. The previous procedure can be iterated straightforwadly for arbitrary numbers $n_{2}$ of second-class particles, which gives

$$
\begin{aligned}
A(k) \Psi\left(k_{1}^{(1)}, \ldots, k_{n_{2}}^{(1)}\right) & =\Lambda^{(A)}(k) \Psi\left(k_{1}^{(1)}, \ldots, k_{n_{2}}^{(1)}\right) \\
& -\sum_{i=1}^{n} \Lambda_{i}^{(A)}(k) \Psi\left(k, k_{1}^{(1)}, \ldots, k_{i-1}^{(1)}, k_{i+1}^{(1)}, \ldots, k_{n_{2}}^{(1)}\right), \\
D(k) \Psi\left(k_{1}^{(1)}, \ldots, k_{n_{2}}^{(1)}\right) & =\Lambda^{(D)}(k) \Psi\left(k_{1}^{(1)}, \ldots, k_{n_{2}}^{(1)}\right) \\
& -\sum_{i=1}^{n} \Lambda_{i}^{(D)}(k) \Psi\left(k, k_{1}^{(1)}, \ldots, k_{i-1}^{(1)}, k_{i+1}^{(1)}, \ldots, k_{n_{2}}^{(1)}\right),
\end{aligned}
$$

where

$$
\begin{aligned}
& \Lambda^{(A)}(k)=\prod_{j=1}^{n} S_{1,1}^{1,1}\left(k_{P_{j}}, k\right) \prod_{i=1}^{n_{2}} \frac{S_{1,1}^{1,1}\left(k, k_{i}^{(1)}\right)}{S_{1,2}^{1,2}\left(k, k_{i}^{(1)}\right)}, \\
& \Lambda^{(D)}(k)=\prod_{j=1}^{n} S_{1,2}^{1,2}\left(k_{P_{j}}, k\right) \prod_{i=1}^{n_{2}} \frac{S_{2,2}^{2,2}\left(k_{i}^{(1)}, k\right)}{S_{1,2}^{1,2}\left(k_{i}^{(1)}, k\right)}, \\
& \Lambda_{j}^{(A)}(k)=\frac{S_{2,1}^{1,2}\left(k, k_{j}^{(1)}\right)}{S_{1,2}^{1,2}\left(k, k_{j}^{(1)}\right)} a\left(k_{j}^{(1)}\right) \prod_{l=1(\neq j)}^{n_{2}} \frac{S_{1,1}^{1,1}\left(k_{j}^{(1)}, k_{l}^{(1)}\right)}{S_{1,2}^{1,2}\left(k_{j}^{(1)}, k_{l}^{(1)}\right)},
\end{aligned}
$$




$$
\Lambda_{j}^{(D)}(k)=\frac{S_{1,2}^{2,1}\left(k_{j}^{(1)}, k\right)}{S_{1,2}^{1,2}\left(k_{j}^{(1)}, k\right)} d\left(k_{j}^{(1)}\right) \prod_{l=1(\neq j)}^{n_{2}} \frac{S_{2,2}^{2,2}\left(k_{l}^{(1)}, k_{j}^{(1)}\right)}{S_{1,2}^{1,2}\left(k_{l}^{(1)}, k_{j}^{(1)}\right)} .
$$

Then $\Psi\left(k_{1}^{(1)}, \ldots, k_{n_{2}}^{(1)}\right)$ is an eigenvector of $T$ with eigenvalue $\Lambda(k)=\Lambda^{(A)}(k)+\Lambda^{(D)}(k)$, i. e.,

$$
\Lambda(k)=\prod_{i=1}^{n} S_{1,1}^{1,1}\left(k_{P_{i}}, k\right) \prod_{\alpha=1}^{n_{2}} \frac{S_{1,1}^{1,1}\left(k, k_{\alpha}^{(1)}\right)}{S_{1,2}^{1,2}\left(k, k_{\alpha}^{(1)}\right)}+\prod_{i=1}^{n} S_{1,2}^{1,2}\left(k_{P_{i}}, k\right] \prod_{\alpha=1}^{n_{2}} \frac{S_{2,2}^{2,2}\left(k_{\alpha}^{(1)}, k\right)}{S_{1,2}^{1,2}\left(k_{\alpha}^{(1)}, k\right)}
$$

if the following conditions, which fix $\left\{k_{1}^{(1)}, \ldots, k_{n_{2}}\right\}$ are satisfied:

$$
\Lambda_{i}^{(A)}(k)+\Lambda_{i}^{(D)}(k)=0, \quad i=1, \ldots, n_{2} .
$$

Using the relations (52), this last condition can be written as

$$
\prod_{j=1}^{n} \frac{S_{1,2}^{1,2}\left(k_{P_{j}}, k_{\alpha}^{(1)}\right)}{S_{1,1}^{1,1}\left(k_{P_{j}}, k_{\alpha}^{(1)}\right)}=\prod_{\beta=1(\neq \alpha)}^{n_{2}} \frac{S_{1,1}^{1,1}\left(k_{\alpha}^{(1)}, k_{\beta}^{(1)}\right) S_{1,2}^{1,2}\left(k_{\beta}^{(1)}, k_{\alpha}^{(1)}\right)}{S_{1,2}^{1,2}\left(k_{\alpha}^{(1)}, k_{\beta}^{(1)}\right) S_{2,2}^{2,2}\left(k_{\beta}^{(1)}, k_{\alpha}^{(1)}\right)}, \quad \alpha=1, \ldots, n_{2},
$$

which concludes the diagonalization of $T(k)$.

Now let us return to our original problem of finding the eigenvalues of the Hamiltonian (6). The Bethe-ansatz equations will be obtained by inserting in (38) the eigenvalues evaluated at $k_{j}$, i. e., $\Lambda\left(k_{j}\right)$, given in (60), with the condition (62). Taking into account that $S_{1,2}^{1,2}(k, k)=0$, we obtain

$$
e^{-i k_{j} N}=(-)^{n-1} \prod_{l=1}^{n}\left(\Xi_{l, j} S_{1,1}^{1,1}\left(k_{l}, k_{j}\right)\right) \prod_{\alpha=1}^{n_{2}} \frac{S_{1,1}^{1,1}\left(k_{j}, k_{\alpha}^{(1)}\right)}{S_{1,2}^{1,2}\left(k_{j}, k_{\alpha}^{(1)}\right)}, \quad j=1, \ldots, n,
$$

with the condition

$$
\prod_{j=1}^{n} \frac{S_{1,2}^{1,2}\left(k_{j}, k_{\alpha}^{(1)}\right)}{S_{1,1}^{1,1}\left(k_{j}, k_{\alpha}^{(1)}\right)}=\prod_{\beta=1(\neq \alpha)}^{n_{2}} \frac{S_{1,1}^{1,1}\left(k_{\alpha}^{(1)}, k_{\beta}^{(1)}\right) S_{1,2}^{1,2}\left(k_{\beta}^{(1)}, k_{\alpha}^{(1)}\right)}{S_{1,2}^{1,2}\left(k_{\alpha}^{(1)}, k_{\beta}^{(1)}\right) S_{2,2}^{2,2}\left(k_{\beta}^{(1)}, k_{\alpha}^{(1)}\right)}, \quad \alpha=1, \ldots, n_{2} .
$$

Writing explicitly the $S$-matrix elements (26) in (63) and (64) we can state that the energies, in the sector with $n_{1}$ first-class particles and $n_{2}$ second-class particles are given by

$$
E=-\sum_{j=1}^{n}\left(\epsilon_{-} e^{i k_{j}}+\epsilon_{+} e^{-i k_{j}}-1\right),
$$

where $\left\{k_{j}, j=1, \ldots, n\right\}$ are given by the solutions of

$$
\begin{aligned}
e^{i k_{j}\left(N-n_{2}\left(s_{2}-s_{1}\right)\right)}= & (-)^{n_{1}-1} \prod_{l=1}^{n}\left[\left(\frac{e^{i k_{j}}}{e^{i k_{l}}}\right)^{s_{1}-1} \frac{\epsilon_{+}+\epsilon_{-} e^{i\left(k_{l}+k_{j}\right)}-e^{i k_{j}}}{\epsilon_{+}+\epsilon_{-} e^{i\left(k_{l}+k_{j}\right)}-e^{i k_{l}}}\right] \\
& \times \prod_{\alpha=1}^{n_{2}} \frac{\epsilon_{+}\left(e^{i k_{j}}-e^{i k_{\alpha}^{(1)}}\right)}{\epsilon_{+}+\epsilon_{-} e^{i\left(k_{j}+k_{\alpha}^{(1)}\right)}-e^{i k_{\alpha}^{(1)}}} \quad(j=1, \ldots, n), \\
e^{i k_{j}\left(s_{2}-s_{1}\right) n} \quad\left(\epsilon_{+}\right)^{n} & \prod_{i=1}^{n} \frac{e^{i k_{j}}-e^{i k_{\alpha}^{(1)}} \frac{\epsilon_{+}+\epsilon_{-} e^{i\left(k_{j}+k_{\alpha}^{(1)}\right)}-e^{i k_{j}}}{\epsilon_{2}}}{(-)^{n_{1}-1}} \quad \prod_{\beta=1}^{n_{2}} \frac{\epsilon_{+}+\epsilon_{-} e^{i\left(k_{\alpha}^{(1)}+k_{\beta}^{(1)}\right)}-e^{i k_{\alpha}^{(1)}} \quad \quad \quad\left(\alpha=1, \ldots, n_{2}\right) .}{\epsilon_{+}+e^{i\left(k_{\alpha}^{(1)}+k_{\beta}^{(1)}\right)}-e^{i k_{\beta}^{(1)}} \quad} \quad
\end{aligned}
$$


It is interesting to observe that in the particular case where $n_{2}=0$ we obtain the Bethe-ansatz equations, recently derived [15], for the asymmetric diffusion problem with particles of size $s_{1}$. Also the case $s_{1}=s_{2}=1$ give us the corresponding Bethe ansatz equations for the standard problem of second class particles. The Bethe-ansatz equations for the fully asymmetric problem is obtained by setting $\epsilon_{+}=1$ and $\epsilon_{-}=0$.

\section{Conclusions and generaliza- tions}

We obtained through the Bethe ansatz the exact solution of the problem of first- and second-class particles diffusing and interchanging positions on the lattice. We show that the solution can be derived in the general case where the particles have arbitrary sizes.

Some extensions of our results can be made. A first generalization is the problem where $M>2$ hierarchical ordered classes of particles (1st, 2nd, ...,Mth classes), with sizes $s_{i}(i=1, \ldots, M)$, besides diffusing on the lattice, interchange positions in such way that particles of higher hierarchical classes see the lower ones in the same way as they see the holes ( 0 class). The allowed processes and the Hamiltonians are simple generalizations of relations (2)-(4) and (6), respectively. The Hamiltonian obtained in a open lattice will be $U_{q}(S U(M))$ symmetric and in the case $s_{1}=s_{2}=\ldots=s_{M}=1$ it is related with the spin $S=\frac{M}{2}$ Sutherland model [17]. The model is certainly integrable and its eigenspectra will be given in terms of $M$-nested Bethe ansatz equations which are generalizations of (66)-(67).

A further quite interesting generalization of our model happens when we consider molecules in one or both classes with size $s=0$. Molecules of size zero do not occupy space on the lattice, having no hardcore exclusion effect. Consequently we may have, at a given lattice point, an arbitrary number of them. The Bethe-ansatz solution presented in the previous section is extended directly in this case (the equations are the same) and the eigenenergies are given by fixing in (65)(67) the appropriate sizes of the molecules. It is interesting to remark that particles of second class with size $s_{2}=0$, contrary to the case $s_{2}>1$, where they "accelerate" the diffusion of the first class particles, now they "retard" the diffusive motion of these particles. The quantum Hamiltonian in the cases where the particles have size zero are written in terms of $\operatorname{spin} s=\infty$ quantum chains.

The Bethe-ansatz equations in the case of the asymmetric diffusion, with particles of unit size [10, 11], or with arbitrary size [15], was used to obtain the finite- size corrections of the mass gap $G_{N}$ of the associated quantum chain. The real part of these finite-size corrections are governed by the dynamical critical exponent $z$, i. e.,

$$
\operatorname{Re}\left(G_{N}\right) \sim N^{-z} .
$$

The calculation of the exponent $z$ for the model presented in this paper, with particles of arbitrary sizes, is presently in progress [24].

\section{Acknowledgements}

This work was supported in part by Conselho Nacional de Desenvolvimento Científico e Tecnológico $\mathrm{CNPq}$ - Brazil and by the Russian Foundation of Fundamental Investigation ( Grant 99-02-17646).

\section{References}

[1] A. A. Lushinikov, Zh. Éksp. Teor. Fiz. 91, 1376 (1986) [Sov. Phys. JETP 64, 811 (1986)], Phys. Lett. A 120, 135 (1987).

[2] G. M. Schültz, J. Stat. Phys. 71, 471 (1993).

[3] F. C. Alcaraz, M. Droz, M. Henkel, and V. Rittenberg, Ann. Phys. (N.Y.) 230, 250 (1994).

[4] F. C. Alcaraz and V. Rittenberg, Phys. Lett. B 324, 377 (1993).

[5] F. C. Alcaraz, Int. J. Mod. Phys. B bf 8, 3449 (1994).

[6] M. D. Grynberg and R. B. Stinchcombe, Phys. Rev. Lett. 74, 1242 (1995).

[7] K. Krebs, M. P. Pfannmüller, B. Wehefritz, and H. Henrichsen, J. Stat. Phys. 78, 1429 (1995).

[8] J. E. Santos, G. M. Schütz, and R. B. Stinchcombe, J. Chem. Phys. 105, 2399 (1996).

[9] M. J. de Oliveira, T. Tomé, and R. Dickman, Phys. Rev. A 46, 6294 (1992).

[10] L. H. Gwa and H. Spohn, Phys. Rev. Lett. 68, 725 (1992), Phys. Rev. A 46, 844 (1992).

[11] D. Kim, Phys. Rev. E 52, 3512 (1995).

[12] D. Kim, J. Phys. A 30, 3817 (1997).

[13] F. C. Alcaraz, S. Dasmahapatra and V. Rittenberg, J. Phys. A. 31, 845 (1998); Physica A 257, 1 (1998).

[14] T. Sasamoto and M. Wadati, J. Phys. A 31, 6057 (1998).

[15] F. C. Alcaraz and R. Z. Bariev, Phys. Rev. E 60, 79 (1999).

[16] C. N. Yang, Phys. Rev. Lett. 19, 1312 (1967).

[17] B. Sutherland, Phys. Rev. B 12, 3795 (1975).

[18] C. Boldrighini, G. Cosimi, S. Frigio, and Nuñes G, J. Stat. Phys. 55, 611 (1989).

[19] P. A. Ferrari, C. Kipnis and E. Saada, Ann. Prob. 19, 226 (1991).

[20] P. A. Ferrari, Prob. Theory Relat. Fields 91, 81 (1992). 
[21] L. A. Takhtajan and L. D. Faddeev, Russ. Math. Surv. 34,11 (1979); V. E. Korepin, I. G. Izergin and N. M. Bogoliubov, Quantum Inverse Scattering Method, Correlation Functions and Algebraic Bethe Ansatz (Cambridge University Press, Cambridge, 1992).

[22] B. Derrida, M. R. Evans, V. Hakim, and V. Pasquier,
J. Phys. A 26, 1493 (1993).

[23] R. J. Baxter, Exactly Solved Models in Statistical Mechanics (Academic Press, New York, 1982).

[24] F. C. Alcaraz and R. Z. Bariev, (to be plublished). 